secretary for fish and wildlife and parks, once stated: "If we are saying that the loss of species in and of itself is inherently bad - I don't think we know enough about how the world works to say that." In 2004, on the day after Bush's re-election, the Fish and Wildlife Service, which Manson oversees, fired the biologist Andy Eller, who had charged that the service was not fulfilling its mandate of protecting the Florida panther, an endangered species. The agency was forced to re-hire Eller after a court ruled in his favour.

Some of the Bush administration's actions have been almost comically incompetent. Last January, for example, George Deutsch, a publicaffairs officer at NASA, tried to prevent the space agency's James Hansen from speaking to the press about the dangers of global warming. Andrew Revkin of The New York Times quickly exposed the attempt to censor Hansen, and it was soon revealed that Deutsch, contrary to what it said on his $\mathrm{CV}$, had never graduated from university. Deutsch resigned and NASA administrator Michael Griffin declared: "It is

\title{
The Universe's quantum monkeys
}

\section{Programming the Universe: A Quantum Computer Scientist Takes On the Cosmos by Seth Lloyd \\ Alfred A. Knopf/Jonathan Cape: 2006. \\ 240 pp. \$25.95/£18.99}

\section{Artur Ekert}

A little less than 14 billion years ago, a huge explosion gave birth to the Universe, and once it sprang into existence, the Universe began computing. The positions, velocities and internal states of every elementary particle, every atom and molecule, indeed every single physical entity register bits of information. Those bits are continually altered by physical interactions that act like sequences of logic gates - given a sufficient supply of bits and enough time, they can compute just about anything that is computable. Thus, the Universe is a computer. It is not a metaphor, it really is. More than that, the fundamental laws of physics that govern any interaction are quantum; hence, the Universe is a huge quantum computer that computes its own behaviour. It started in a very simple state initially, but in time, as the number of computational steps increased, the computing quantum Universe spun out more complex patterns, including galaxies, stars and planets, and then life, humans, you and me, and Seth Lloyd and his book Programming the Universe.

Like many other good stories of this type, Lloyd's book will puzzle and even irritate as much as it persuades. Lloyd writes in a lively style, weaving jokes and personal anecdotes into more technical narrative. He shares his views on cosmology, computation, quantum not the job of public-affairs officers to alter, filter or adjust engineering or scientific material produced by NASA's technical staff."

But the damage caused by the Bush administration's contempt for scientific facts is no was re-elected, "reality" - especially the reality States invaded on the basis of erroneous technical claims - has humbled his administration. In November, as the death toll in Iraq surged, voters handed the reins of power in Congress to the Democrats. In December, the Iraq Study Group, headed by the Bush family friend James Baker, issued a scathing critique of the US occupation of Iraq. Bush's approval rating has sunk to one of the lowest levels ever recorded. The declaration of that Bush official - "We tragic irony.

John Horgan is director of the Center for Science Writings at the Stevens Institute of Technology, Hoboken, New Jersey. His most recent book is Rational Mysticism. laughing matter. In the two years since Bush of Iraq, which, Shulman points out, the United create our own reality" - has now taken on a physics, complexity, sex, life, the Universe and all that, and he does it well. Despite this proliferation of topics, the main message stands out and is reiterated several times - the Universe is a quantum computer programmed by quantum fluctuations, and the computational capability of the Universe explains how complex systems can arise from fundamentally simple physical laws.

Lloyd tells the story of the evolving Universe in terms of interplay between energy and information. In the conventional history of the origin and the evolution of the Universe, the story usually told by cosmologists and astronomers, energy plays the central role. First there was a singularity and there was no past for it to emerge from. Then expansion. As the Universe expanded, it cooled down and various forms of matter condensed out because the disruptive thermal energy gradually dropped below the binding energies that hold constituent parts of protons, nuclei and atoms together. Tiny quantum fluctuations made some regions of the Universe slightly denser, and gravity amplified this effect, which resulted in gas clouds, stars and galaxies. Stars exploding to supernovae produced heavier elements, then our Sun and Solar System formed, and about 4 billion years ago, life emerged on Earth. But this story leaves many questions unanswered. How did life arise? Why is the Universe so complex? Could such complexities have arisen from total randomness?

Now, enter computer science. Algorithmic information theory shows that there are short, random-looking programs that can cause a computer to produce complex-looking outputs. Lloyd illustrates this with a popular story attributed to the French mathematician Émile Borel. Imagine a bunch of monkeys typing randomly into typewriters. Given enough time, it is certainly possible that one of these monkeys will type the first million digits of $\pi$ or the first act of Hamlet. Possible, but very unlikely. Now, take the typewriters away and give the monkeys computers that recognize any random inputs not as text but as a computer program. When the computers try to execute random programs, most of the time they will crash or generate garbage, but every now and then just a few lines of random code typed by monkeys will give interesting outputs - for example, the successive digits of $\pi$, or intricate fractals. Or perhaps much more interesting patterns if the computer is the Universe itself.

This vision of a computational Universe is

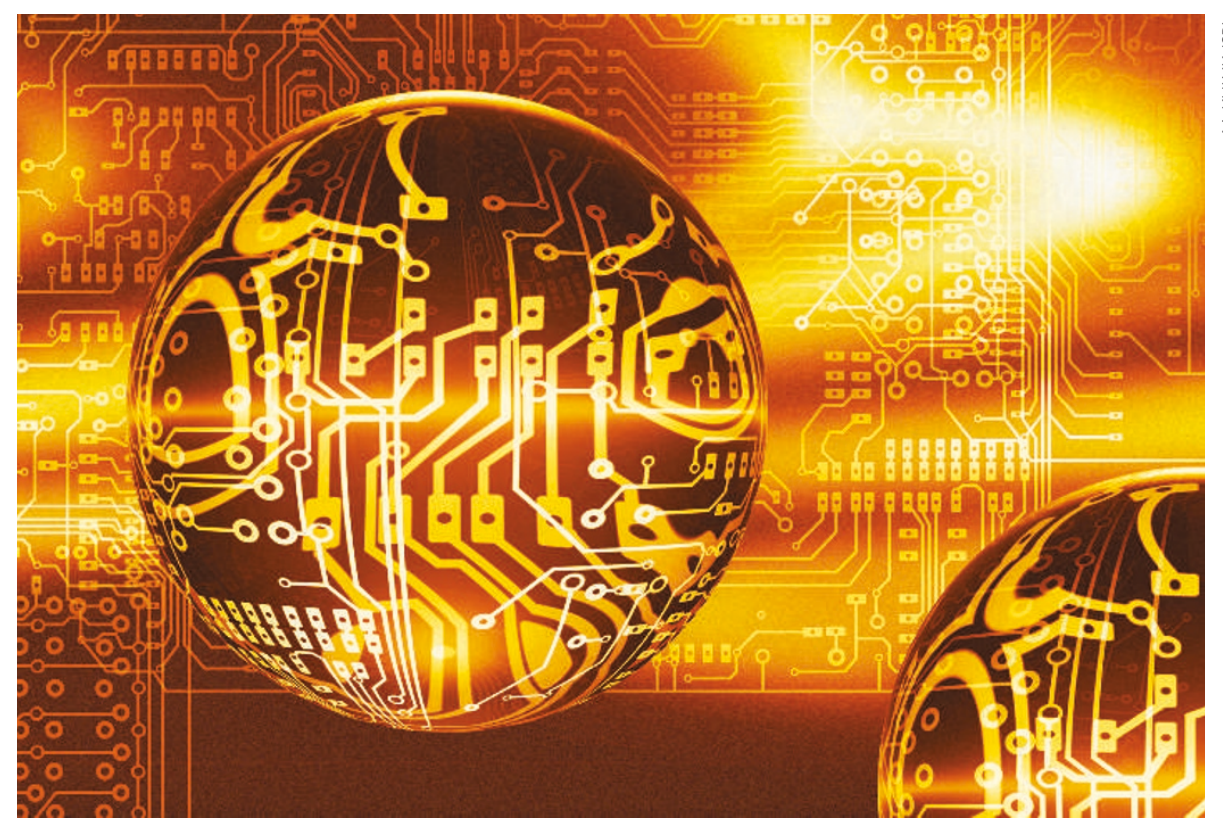

Get with the program: the Universe, it turns out, is actually a giant quantum computer. 
not new: it was proposed in the 1960s by Konrad Zuse and Ed Fredkin, and revived more recently by Stephen Wolfram. However, unlike his predecessors, Lloyd stresses the quantum nature of computation. This distinction is important because, to the best of our knowledge, it seems impossible to simulate the evolution of a quantum system in an efficient way on a classical computer.

A classical computer simulation of quantum evolution typically involves an exponential slowdown in time. This is because the amount of classical information needed to describe the evolving quantum state is exponentially larger than that needed to describe the corresponding classical system with similar accuracy. However, instead of viewing this intractability as an obstacle, today we regard it as an opportunity - if that much computation is needed to work out what will happen in a quantum multi-particle interference experiment, then the very act of setting up such an experiment and measuring the outcome is equivalent to performing a complex computation. Since
Richard Feynman and David Deutsch pointed out this opportunity in the 1980s, the hunt has been on for interesting things for quantum computers to do, and at the same time, for the scientific and technological advances that could allow us to build quantum computers. The field is flourishing, and Lloyd provides a good popular introduction to the subject. However, he does not stop at the level of building quantum computers, he takes on the biggest quantum computer there is - the Universe.

The Universe is a quantum computer, and quantum mechanics supplies the Universe with 'monkeys' in the form of ubiquitous random quantum fluctuations - the same fluctuations that provided the seeds of galaxy formation and of all that followed. The Universe has pockets of complex behaviour because, Lloyd claims, the monkeys have been working very hard. He estimates that the visible Universe, programmed by quantum fluctuations, has performed about $10^{122}$ operations on $10^{92}$ quantum bits. No wonder we are here!

I think this is a delightful book, but some parts are patchy and many details are brushed under the carpet. For example, anyone trying to work out numerical estimates of the physical limits to computation or the computational capacity of the Universe is much better off consulting Lloyd's original paper on the subject (see Nature 406, 1047-1054; 2000). It is clear that Lloyd has forsaken accuracy for snappiness in several places, but then this is a popular exposition.

Seth Lloyd is a good storyteller, but is the story convincing? Well, I was convinced, but when I tried a nice line from the book "programmed by quanta, physics gave rise to chemistry and then to life, programmed by mutation and recombination, life gave rise to Shakespeare, programmed by experience and imagination, Shakespeare gave rise to Hamlet" - on a colleague of mine, an English literature fellow, he only shook his head in disbelief and walked away.

Artur Ekert is at the Mathematical Institute, University of Oxford, UK, and the National University of Singapore.

\section{Cover story}

\section{Skin: A Natural History \\ by Nina G. Jablonski \\ University of California Press: 2006. \\ 290 pp. \$24.95, £15.95}

\section{John Galloway}

Biology is a historical science. Ask a 'why?' question about biology, as Nina Jablonski keeps doing in her book Skin, and you invite an evolutionary answer. She also tells us everything we might want to know about skin; perhaps more than some people want to know. She then goes on to take informed guesses as to why skin is the way it is and, by implication, why it is not like something else. Skin's appearance, its form and function, questions of how and why it works, and sometimes doesn't, have been thrashed out over a billion or so years at - to borrow her words - the "negotiating table of evolution".

For Alexander Pope, the "proper study of mankind" may have been "man", but Jablonski, as befits a modern biologist, thinks otherwise. Understanding starts, and possibly finishes, with comparisons, between humans and our biological relatives and neighbours, both near and not-so-near. We may share virtually all our genes with chimpanzees, but those we don't share are responsible for a lot of differences, reproductive, linguistic and cognitive. Skin genes, for example, which are responsible among other things for colour, body hair and the number of sweat glands, may well explain why chimps are still confined to African jungles, whereas we, their closest relatives, have already been to the Moon.

Skin is not just about biology, but also the

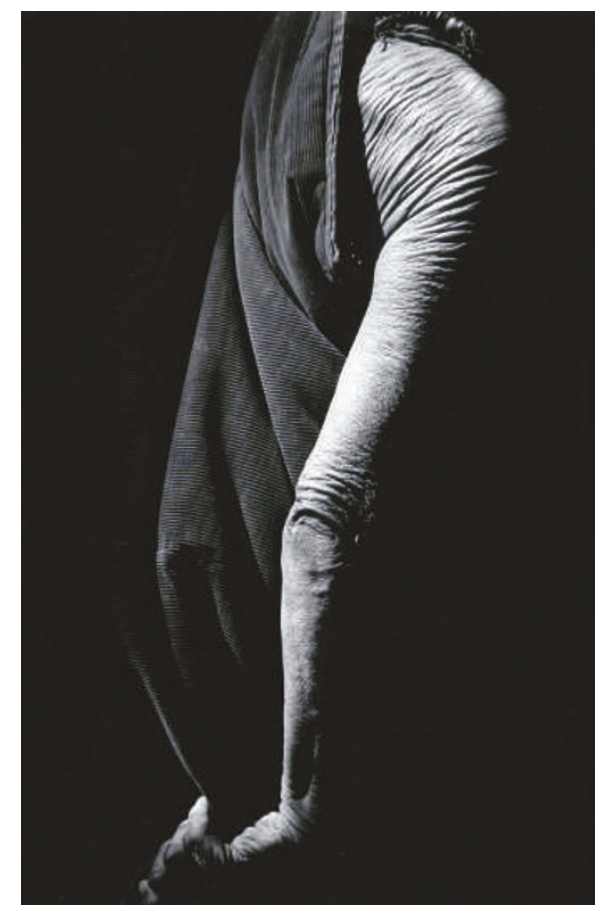

The skin's characteristics have been thrashed out at "the negotiating table of evolution".

way we live. Our skin is the visible, immediate personal territory where biology most obviously gives way to culture. Jablonski quotes Franz Kafka, who had the right idea, viewing the skin as "not only a garment but also a straitjacket and fate". People go to a lot of trouble and expense to alter their appearance and change their fate. From war-paint and cosmetics to tanning, bleaching, tattooing, ritual scarring, botox, body piercing and 'nipping and tucking', there is someone making money out of it. And it does not necessarily have to stop just because someone is dead, as some enterprising Ancient Egyptian undertaker realized.

Some forms of personal make-over and disguise teach a salutory lesson: that culture comes at a biological price, paid from the genetic legacy bequeathed you by evolution. You are, let's say for the sake of argument, a fair-skinned northern European. But it has become the thing to show off a nice tan (Jablonski fingers fashionista Coco Chanel as the perpetrator of this particular vanity), and that means lying about without clothes in hot sun in latitudes rather nearer the Equator. The trouble is, the reason you are fair is a good historical one, indeed a matter of life and death for your ancestors in the Europe of 50,000 or so years ago. And that fact has implications for modern day Sun-worshippers, some of whom discover that mortality still starts with the skin.

At the core of Jablonski's theme is the skin's ability to multi-task: it protects, controls temperature, senses the world around you, and shows people how you really feel, as opposed to what you choose to tell them. But skin is also a chemical factory, fuelled in part by solar radiation. It manufactures vitamin $\mathrm{D}$, without which you can neither extract calcium from your diet nor incorporate it in your bones posing something of a challenge to survival. Here's an evolutionary conundrum. Ultraviolet light, which damages DNA directly and also destroys the folic acid essential for its synthesis, is, ironically, the energy source needed to make vitamin D. In equatorial Africa, our ancestral home, evolution engineered a nice compromise that allowed humans to leave the sheltering forest canopy and begin global colonization. Melanins that absorb ultraviolet 